\title{
How to empower patients, and involve the public
}

\begin{abstract}
Individual empowerment and Patient and Public Involvement are a key focus for contemporary NHS policy in a quasi-market health economy concerned to emphasise patient decision making and representation as a feature of a customer focused NHS. Such language suggests a rebalancing of power in the nurse-patient relationship. The reality is it masks the wider issues of power and control in a complex health service of professional agendas and leadership, government targets and a business culture. Nevertheless, and despite these ideological and organisational constraints, there are ways in which nurses can facilitate aspects of individual patient empowerment and Patient and Public Involvement.
\end{abstract}




\section{How to empower patients, and involve the public}

\section{Introduction}

Ostensibly the language of patient empowerment and Patient and Public Involvement (PPI) signal a rebalancing of power in the nurse-patient relationship. A shift from topdown, hierarchical and nursing led practice to a more 'bottom - up' patient and process based inclusive way of working. But this simplifies the complexity of empowering patients and enabling PPI in a centrally and target/contract driven NHS of multi layered nursing, management, business and professional agendas. This article reviews the concepts, language, meaning and reality of patient empowerment and PPI. Suggested indicators are reviewed together with both the challenges to implementing and ways of measuring impact. The article concludes with examples of how nurses can facilitate empowerment and PPI within contemporary NHS structures.

\section{Background}

The language of patient empowerment, PPI and related terminology are now embedded, and have been for some years, in NHS policy (for example DoH 2001, 2002, 2003, 2004, 2010, 2012). The emphasis is on active decision making by patients and their representatives and is the NHS equivalent of the customer focused language of commerce and the consumer culture. The underpinning principles are consumer autonomy and championing the consumer voice in an NHS market increasingly concerned to facilitate patient choice (Martin 2009). As such they are underpinned by consumer watchdog initiatives such as Patient advice and liaison services (DoH 2002). 
PPI, also known as 'user involvement' (Fudge, Wolfe and Mckevitt 2008) and 'service user involvement' (Cotterell et at 2010) is an ill-defined and contested concept that lacks an established theory base and a common set of meanings. However, it is clear that PPI differs from the 'individual action perspective' (Piper 2009) of patient empowerment. The focus for the latter is the development of an interpersonal, two-way supportive nurse-patient relationship (Piper 2009, 2010) including a shift of power from nurses to individual patients.

PPI is concerned to champion the voice of patient groups via their representatives in the management corridors of the NHS. To this end statutory obligations have been placed on NHS Trusts to ensure PPI in all strategic decision making (Cotterell et at 2010). This is seen as a way to improve the quality of services (Martin 2009) by 'redrawing' (Tritter and McCallum 2006) or ‘transforming' (Tritter and McCallum 2006, Fudge, Wolfe and Mckevitt 2008) lay-professional power relations.

PPI then is about services being shaped by patient groups rather than the priorities of NHS Trusts (Bradshaw 2008, Martin 2009) and about making NHS managers, nurses and other clinicians more responsive to collective patient identified needs (Attree et al 2010). It also redefines the state-citizen relationship (Tritter and McCallum 2006) by going beyond the patient as consumer exercising individual choice to a form of ‘participatory citizenship' (Martin 2009).

In summary then, individual patient empowerment and PPI share a common concern for patient driven decision-making (Brooks 2008) and a more 'bottom-up' patient or patient representative defined health service agenda. They seek to rebalance decision 
making in favour of individual or patient group perception of need rather than nurse led, evidence based and disease focused behaviour change. Both help close the 'social distance' (Beattie 1991) between nurse and patient with the process of intervention as important as impact measures.

\section{Patient empowerment and PPI impact}

There are a number of indicators of successful impact of individual patient empowerment. These include a 'supportive and trusting' (Piper 2010 p174) nursepatient relationship, partnership working, increased patient understanding about treatment options and active and informed individual patient decision making and choice (Piper 2009). Piper $(2009,2010)$ also identified advocacy and helping patients to develop coping strategies and a realistic perception of their situation as impact indicators of empowerment.

The impact of PPI in the NHS needs to be understood more fully. It is complicated by the context and breadth of variables challenging the reliability of tools used (Attree et al 2010, Mockford et al 2012). However, Mockford et als (2012) systematic review did identify areas of positive impact of PPI on healthcare. These included influencing service location, access and infrastructure design, change at institutional and service levels and better health professional - patient and patient -patient dialogue.

In terms of health research, Brett et al (2012) found that the unique perspective offered by PPI, particularly when service users are in the role of research partner, can have a positive impact on all aspects of the research process. This includes the relevance and fitness for purpose of the study commissioning, findings and 
dissemination. This is further enhanced by the cultural awareness PPI offers, the potential for improving research design and data collection tools and the identification of areas of inquiry that might otherwise be overlooked. Staniszewaska et al (2011) add that further impact could be achieved by PPI in research by joint working on developing ways to measure impact.

\section{Patient empowerment and PPI: rhetoric or reality}

The reality of patient empowerment and PPI can only be assured when a number of questions have been answered. In relation to individual empowerment for example, Bradshaw (2008) asks whether a market style, consumer culture can be applied to patient choices. He wonders if patient's have the knowledge and skills to make rational judgements about what services to use and how to access them. Bradshaw (2008) also questions whether choice can be fulfilled in a cash strapped NHS and how those that want an active, as opposed to a passive, role in their care are going to be identified. For Tritter and McCallum (2006), while choices made must shape services provided changes of this nature are indicative of a reframing of clinical relationships to one of customer/consumer - vendor. Whether the latter will automatically lead to better quality service provision remains to be seen (Fudge, Wolfe and Mckevitt 2008)?

Key to facilitating individual patient empowerment is building a supportive and trusting nurse-patient relationship to create the right climate for informed decision making and choice (Piper 2010). This requires more than a slight modification to traditional lay-professional relations (Piper 2010). The power nurses have over the empowerment process itself (if, when, how far to take it) also needs to be addressed. 
However, where this is achieved it is important to remember that informed decision making might well result in empowered patients rejecting/ignoring the advice of the nurse or changing their minds. Patient choice is influenced by such things as a wider personal agenda, life context, money and spiritual and cultural beliefs (Piper 2009, Whitney, McGuire and McCullough 2004, Anderson and Funnell 2010) and not just by pathological, therapeutic (Aujoulat, d'Hoore, Deccache 2007) or evidence based priorities. Conversely informed decision making is little more than rhetoric when undermined by the use of the language of clinical research and evidence based outcomes by nurses thus widening rather than narrowing the nurse-patient social gap.

Further to this it needs to be understood that while the rhetoric of patient empowerment and PPI can be suggestive of a developing consumer culture and devolution of power to patients, in practice this may be restricted by a number of factors. For example, Fudge, Wolfe and Mckevitt (2008) found that the nature of PPI was defined and initiatives were led by 'health professionals' who also acted as gate keepers to involvement. This might help explain the uncertainty many patients feel about the terms of reference and expectations of what is to be achieved when joining service user groups (Cotterell et at 2010). Fudge, Wolfe and Mckevitt (2008) also found how even activities of minimal involvement such as patient satisfaction surveys are labelled as PPI. They noted that where PPI did take place it tended to be restricted to 'low tech' settings.

In addition, for Fudge, Wolfe and Mckevitt (2008) exactly how the statutory obligations for consultation and representation of the patient voice at a strategic level within the NHS are to be achieved remains unclear. An added complication for PPI is 
how to ensure genuine representation for all rather than particular sections of society (Bradshaw 2008, Martin 2009) and how to identify, unravel and resolve any competing agendas (Martin 2009). PPI is also inhibited if nurses lack the skills and preparedness to interact with patients from a position of equality (Brooks 2008) and, in effect, if nurses fail to engage with the process (Cotterell et al 2010).

Thus, more evidence is needed on both PPI and patient empowerment as follows. Firstly on the patient advantage (Cotterell et at 2010) and cost effectiveness of PPI (Fudge, Wolfe and Mckevitt 2008, Attree et al 2010, Mockford et al 2012). Secondly, on whether nurses feel sufficiently empowered themselves to empower others. Thirdly, in light of Bradbury-Jones, Sambrook and Irvine's (2007) findings, how the use of power impacts on student nurses experience of empowerment and disempowerment in practice settings.

Notwithstanding the above, it would seem that some patients are either not interested (Salmon and Hall 2004, Lewin and Piper 2007) or are more concerned about restoration of function than issues relating to empowerment (Loft, McWilliam and Ward-Griffin 2003). Lewin and Piper (2007) found individual patient empowerment to be of minimal interest to acutely ill patients who were happy to be passive recipients of care based on the confidence they had in the nursing and medical staff. Most patients readily surrendered power and patient satisfaction was high.

Finally, it must not be forgotten that in a financially constrained environment opportunity cost (Piper 2009) becomes more of an issue. The reality is that the high 
price treatment chosen by one patient might well be at the expense of treatment for another (Hewitt-Taylor 2004).

\section{Patient empowerment, PPI and nursing: rhetoric or reality?}

Nurses can start to make individual patient empowerment a reality in a number of ways. For example, by reflecting on their current nurse-patient interpersonal style and professional/ideological stance and by actively moving towards the empowerment indicators discussed earlier. By looking for every opportunity to reduce nursing control over decision making and identifying opportunities to facilitate an increase in patient influence in all areas of nursing practice and organisation. This requires existing communication skills to be used to good effect to illicit and enable patient choice, informed decision making and advocacy on behalf of patients (Piper 2009).

But the bigger challenge is the contribution of nursing to making PPI a reality. The contested meaning of PPI, the still emerging theory base and definitive measures of impact and vested interests in retaining the status quo mean it can be difficult to translate policy into action (Forbat et al 2009). Nevertheless, nursing can and must grapple with this and develop strategies for engagement. The dangers of nursing resistance are professional marginalisation as PPI increasingly comes to influence NHS decision making (Brooks 2008).

To contribute effectively to PPI nurses will need to enable the development of nursepatient representative relationships of greater reciprocal power and to acknowledge 'patient experiences and expertise' (Brooks 2008, p4). Partnership working will need 
to be adopted as the dominant mode of intervention and obstructive institutional and professional barriers challenged.

Nurses can also move beyond rhetoric by encouraging PPI, for example, in cancer patients. This can help to reshape the patient's perception of their diagnosis in a positive way and help them separate the pathology from their identity (Cotterell et at 2010). It can reduce the sense of isolation while boosting hope, 'confidence,' 'belonging,' inspiration and the feeling of making a positive contribution. Some patients also gain from the positive role modelling of fellow cancer survivors (Cotterell et at 2010) and the experience of illness of one patient can help others in a similar situation (Mockford et al 2012).

Nurses can raise awareness about PPI support and self-help groups. Such groups can provide peer support networks for patients, partners and carers. They can provide a conduit for what Mockford et al (2012) refer to as patient-patient and patientprofessional dialogue. From a PPI perspective this can provide a focal point for lobbying for resources, services and service development (Piper 2009).

\section{Conclusion}

Patient empowerment and PPI are part of a modernising culture in NHS policy, language and service delivery that invoke notions of consumerism, openness, transparency and democratic changes to the NHS culture. Nonetheless, they remain ill defined, contested concepts with variations in practice, an evolving evidence base and with tools for measuring impact and effectiveness still developing. Challenges to the rebalancing of inequalities in nurse-patient power include scientifically driven evidence based practice and by a related professional culture and language. In 
dictating the process and context of health care they widen rather than narrow the nurse-patient social and knowledge based gaps. But despite this, and while challenges to these ideological, structural and professional barriers evolve, in reality there is room for nurses to contribute to real, positive individual patient empowerment and PPI impact. Nurses can actively set out to equalise the nurse-patient relationship and facilitate individual patient empowerment and PPI. To achieve this an appropriate mind set needs to be adopted together with the use and further development of strategies and modes of intervention akin to those described herein.

\section{Implications for nursing research and education}

Individual patient empowerment is well discussed in the nursing and health care literature and is not a new concept in the same way as PPI. Thus nursing research in consultation with patients and their representatives needs to clarify and contextualise the ill-defined and contested meaning of PPI in particular and identify specific indicators for nursing practice and ways of enabling the process. Added to this, nursing academics need to ensure that patient empowerment and PPI are embedded in the culture, teaching and learning strategies of nurse education and not just in the rhetoric of curriculum documentation. Student nurses need to understand and feel able to develop practice in line with this contemporary health care agenda and to do this need to become and feel empowered. Lastly, if NHS Trusts are serious about individual patient empowerment and PPI then it must be integrated into continuing professional development (CPD) training. 


\section{References}

Anderson RM, Funnell MM (2010) Patient empowerment: myths and misconceptions. Patient Education and Counselling,79, 277-282.

Attree P, Morris S, Payne S, Vaughan S, Hinder S (2010) Exploring the influence of service user involvement on health and social care services for cancer. Health Expectations, 14, 48-58.

Aujoulat I, d'Hoore W, Deccache A (2007) Patient empowerment in theory and practice: polysemy or cacophony? Patient Education and Counselling, 66, 13-20.

Beattie A (1991) Knowledge and Control in Health Promotion: A test case for social policy and social theory. In: Gabe J, Calnan M, Bury M, eds. The Sociology of the Health Service, London: Routledge, 162-202.

Bradbury-Jones C, Sambrook S, Irvine F (2007) The meaning of empowerment for nursing students. Journal of Advanced Nursing, 59, 4, 342-351.

Bradshaw P L (2008) Service user involvement in the NHS in England: genuine user participation or a dogma-driven folly?

Brett $\mathbf{J}$ et al (2012) Mapping the impact of patient and public involvement on health and social care research: a systematic review. Health Expectations, p1-14.

Brooks F (2008) Nursing and public participation in health: an Ethnographic study. International Journal of Nursing Studies, 45, 3-13.

Cotterell P, Harlow G, Morris C et al (2010) Service user involvement in cancer care: the impact on service users. Health Expectations, 14, 159-169.

Department of Health (2001) The expert patient: a new approach to chronic disease management for the $21^{\text {st }}$ century. London: Department of Health.

Department of Health (2002) Patient advice and liaison service. London: Department of Health.

Department of Health (2003) Strengthening Accountability: Involving Patients and the Public. Practice Guidance. Section 11 of the Health and Social Care Act 2001. London: Department of Health,.

Department of Health (2004) Patient and pubic involvement in health: The evidence of policy implementation. London: Department of Health.

Department of Health (2010) Equity and Excellence. Liberating the NHS

Department of Health (2012) Choice Framework. www.dh.gov.uk/publications Accessed March 20, 2013. 
Forbat L, Hubbard G, Kearney N, (2009) Patient participation: Patient and Public Involvement: Models and Muddles. Journal of Clinical Nursing. 18, 2547-2554.

Fudge N, Wolfe CDA, Mckevitt C (2008) Assessing the promise of user involvement in health service development: ethnographic study. BMJ, 336,7639, 313-317.

Hewitt-Taylor J (2004) Challenging the balance of power: patient empowerment. Nursing Standard, 18, 22, 33-37.

Lewin D, Piper SM (2007) Patient empowerment within a coronary care unit. Intensive and Critical Care Nursing, 23, 81-90.

Loft M, McWilliam C, Ward-Griffin C (2003) Patient empowerment after total hip and knee replacement. Orthopaedic Nursing, 22, 1, 42-47.

Martin, GP (2009) Whose health, whose care, whose say? Some comments on public involvement in the NHS commissioning arrangements. Critical Public Health, $19,1,123-132$.

Mockford C, Stanizewska S, Griffiths F, Herron-Marx S (2012) The impact of patient and public involvement on UK NHS health care: a systematic review. International journal for Quality in Health Care, 24, 1, 28-38.

Piper SM (2009) Health Promotion for Nurses: Theory and practice. Abingdon: Routledge.

Piper SM (2010) Patient empowerment: Emancipatory or technological practice? Patient Education and Counselling, 79, 173-177.

Salmon P, Hall GM (2004) Patient empowerment or the emperor's new clothes? Journal of the Royal Society of Medicine; 97, 53-56.

Staniszewaska S et al (2011) Developing the evidence base of patient and public involvement in health and social care research: the case for measuring impact. International Journal of Consumer Studies, 35, 28-632.

Tritter J, McCallum A (2006) The snakes and ladders of user involvement: Moving beyond Arnstein. Health Policy, 76, 156-168.

Whitney SN, McGuire AL, McCullough LB (2004) A typology of shared decision making, informed consent and simple consent. Annals of internal medicine, 140, 5459. 